\title{
The Infectivity of Pulmonary Tuberculosis in Korean Army Units: Evidence from Outbreak Investigations
}

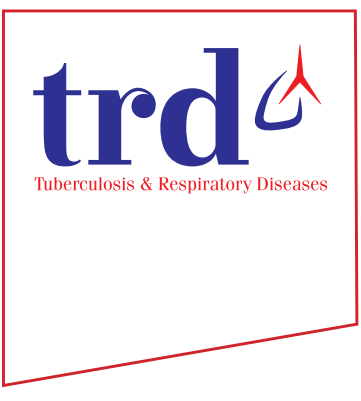

\author{
Chang-gyo Yoon, M.D., M.P.H. ${ }^{1,2}$ i⿺ Dong Yoon Kang, M.D., Ph.D. ${ }^{3,4,5}$, Jaehun Jung, M.D., M.Sc. ${ }^{1,6}$, \\ Soo Yon Oh, R.N., M.Sc. ${ }^{7}$, Jin Beom Lee, R.N., M.Sc. ${ }^{7}$, Mi-Hyun Kim, B.Sc. ${ }^{7}$, Younsuk Seo, B.Sc. ${ }^{7}$ \\ and Hee-Jin Kim, M.D., M.Sc. ${ }^{7}$ (iD
}

${ }^{1}$ Department of Preventive Medicine, Armed Forces Medical Command, Seongnam, ${ }^{2}$ Preventive Medicine Program, Graduate School of Public Health, Seoul National University, Seoul, ${ }^{3}$ International Peace Supporting Standby Force, Ministry of National Defense, Seoul, ${ }^{4}$ Department of Preventive Medicine, Seoul National University College of Medicine, Seoul, ${ }^{5}$ Drug Safety Monitoring Center, Seoul National University Hospital, Seoul, ${ }^{6}$ Department of Preventive Medicine, Korea University College of Medicine, Seoul, ${ }^{7}$ Korean Institute of Tuberculosis, Seoul, Korea

Background: Tuberculosis (TB) causes substantial health burden to the Korean military. This study aims to assess the impact of infectious TB cases on close and casual contacts in the Korean Army settings based on contact investigation data. Methods: Six Army units with infectious TB cases from September 2012 to May 2013 were enrolled in the study. We analyzed the clinical data from close and casual contacts screened using the tuberculin skin test (TST) and QuantiFERON-TB Gold In-Tube to identify latent tuberculosis infection (LTBI) cases. For the control group, 286 military conscripts with no reported TB exposure were tested by TST only.

Results: Of the 667 contacts of index cases, LTBI cases identified were as follows: $21.8 \%$ of close contacts of smearpositive cases (71/326), 8.5\% of casual contacts of smear-positive cases (26/305), and 2.8\% of close contacts of smearnegative cases (1/36). In the control group, $16.8 \%$ showed positivity in TST. In a multivariate analysis, having stayed in the same room or next room with TB patients was identified as a risk factor of LTBI.

Conclusion: Using the data from TB contact investigations in the Korean Army units, we found an overall LTBI rate of 14.7\% among the contacts screened. This study demonstrates that contacts living in the same building, especially the same room or next room, with TB patients are at a high risk of acquiring LTBI, serving as additional evidence for defining close and casual contacts of a TB patient with regard to Army barrack settings.

Keywords: Tuberculosis; Asian Continental Ancestry Group; Disease Outbreaks; Military Personnel; Korea

Address for correspondence: Hee-Jin Kim, M.D., M.Sc.

Korean Institute of Tuberculosis, 168-5 Osongsaengmyeong 4-ro,

Heungdeok-gu, Cheongju 28158, Korea

Phone: 82-43-249-4900, Fax: 82-43-249-4989

E-mail: hatchingbird@yahoo.co.kr

Received: Oct. 2, 2018

Revised: Mar. 24, 2019

Accepted: May. 2, 2019

Published online: May. 31, 2019

(c) It is identical to the Creative Commons Attribution Non-Commercial License (http://creativecommons.org/licenses/by-nc/4.0/).

Copyright $\odot 2019$

The Korean Academy of Tuberculosis and Respiratory Diseases.

\section{Introduction}

Tuberculosis (TB) is a persistent public health concern in the Republic of Korea as the incidence rate of TB remains to be 55.0 per 100,000 population in $2017^{1}$. TB was the leading cause of death among infectious diseases in 2016, resulting in 2,186 deaths ${ }^{2}$. With enhanced efforts to decrease the TB incidence rate observed in 2010 by half in 2020 , the estimated incidence rate of $\mathrm{TB}$ has been decreasing ${ }^{1,3}$. As the overall incidence rate of $\mathrm{TB}$ decreases, however, more sporadic TB outbreak cases occurring in small groups such as in schools, nurseries, healthcare settings, prisons, and military barracks and the spread of TB through hospital settings have become a 
public health concern ${ }^{4-6}$.

No study has revealed an age- and sex-standardized comparison between TB incidence in the military population and in the general population of Korea, but a study reported that the crude incidence rate of pulmonary TB in the enlisted soldiers was similar to that of the general population in the same age group from 2001 to 2004, ranging from 63.1 to 89.3 per 100,000 population and from 62.0 to 94.8 per 100,000 population, respectively ${ }^{7}$. Due to collective living environments, the military population is more vulnerable to infectious respiratory and enteric diseases including TB than is the civilian population $^{8-14}$. To control the spread of TB in military units, the Armed Forces Medical Command (AFMC) and the Korea Centers for Disease Control and Prevention (KCDC) have been implementing TB control programs that include proper case management, which includes the identification and preventive chemotherapy of latent tuberculosis infection (LTBI) cases among contacts of index cases ${ }^{15}$.

To date, a number of studies were conducted to assess the impact of infectious TB cases in the Korean military settings ${ }^{5,10,16}$. A study conducted in 2006, which used interferon $\gamma$ releasing assay (IGRA), identified that $14.3 \%$ of the 175 contacts of active pulmonary TB patients had LTBI. Moreover, long exposure and laryngeal TB of an index patient were found to be the risk factors of $\mathrm{LTBI}^{11}$. Choi et al. ${ }^{16}$ showed that the close contacts of a smear-positive pulmonary TB case demonstrated an adjusted odds ratio (AOR) of 1.7 during tuberculin skin test (TST) when compared with the non-exposed group. A study conducted in military hospitals demonstrated that military health workers providing TB patient care for greater than 1 year were likely to have LTBI with an AOR of $2.27^{5}$. A study that reviewed the TB investigation database presented that 127 outbreak investigations conducted in 145 index cases in military or police units found 30 new patients and 1,085 LTBI, which accounted for $11.6 \%$ of all contacts screened $(1,085 / 9,335)^{17}$.

Given that the previous studies were conducted only for a single military unit or military hospitals, this study aims to measure the transmission of pulmonary TB cases to close and casual contacts in multiple Korean Army units based on contact investigation data from 2012 to 2013.

\section{Materials and Methods}

\section{Data collection}

In order to enroll TB outbreak cases in the Army units from September 2012 to May 2013, the Army headquarter and subordinate commands were contacted by the Korean Institute of Tuberculosis (KIT). With the information provided, a team from the KIT with military medical epidemiologists visited the units for further investigations. In identifying close and casual contacts, we referred to the National TB Management Guideline and the TB Contact Investigation Guideline developed by the AFMC and KCDC, which define a close contact as a person who has stayed in the same space with a pulmonary TB patient greater than three months at the point of investiga$\operatorname{tion}^{3,15}$. The guidelines define a casual contact as someone who has not shared a space for living but has contacted a pulmonary TB patient by any chance. We therefore categorized military personnel of the units under investigation into close and casual contacts with the following definition: close contacts, having lived with index cases in the same floor in the barrack, and casual contacts, having lived in different barracks but had possible contacts when using mess hall and other attached facilities.

During the study period, we enrolled six Army units, 829 close and casual contacts and 320 control subjects: 273 were close contacts of smear-positive index cases, 520 were casual contacts of smear-positive index cases, 36 were close contacts of smear-negative index cases, and 320 were the control group without any reported TB exposure during the military service. A total of 953 subjects provided complete information for analysis: 326 close contacts of smear-positive index cases, 305 casual contacts of smear-positive index cases, 36 close contacts of smear-negative index cases, and 286 of the control group. The study did not include any subjects with human immunodeficiency virus (HIV) infection as individuals with HIV infection will not be conscripted for military services.

\section{Study protocol}

With the clinical information of index cases obtained using written informed consent forms, we reviewed their medical records to identify the key variables such as age, sex, the presence of a cavity lesion in the lung by chest X-ray (CXR), the results of initial sputum smear, sputum culture, presentation of pulmonary TB-related symptoms, and the total duration of symptoms.

In this study, we adopted the military TB control guideline's ${ }^{15}$ definition of LTBI stating that an LTBI case is a subject showing a positive result in the QuantiFERON-TB Gold InTube (QFT-GIT) or obtaining a positive result at the second round of TST after an initially negative result in the first round of TST among close and casual contacts of an index TB case. All close and casual contacts underwent CXR to assess for the presence of active pulmonary TB lesions. After confirming the absence of active lesions, we screened close and casual contacts by TST and QFT-GIT to identify LTBI cases $^{3}$. In consideration of military training schedules and transfer, the research team and military medical epidemiologists decided to conduct the first round of TST and QFT-GIT at the first contact with the subjects to save time and efforts. For the control group, soldiers without any prior reported exposures to $\mathrm{TB}$ during the military service or active TB history underwent 
TST only to calculate for the relative risk. In order to analyze the risk factors of LTBI among close and casual contacts, we collected demographic information such as age, sex, and bacillus Calmette-Guerin (BCG) vaccination scar in the subject's shoulder, and the living places of contacts in line with index cases.

One of the guidelines set by the National Immunization Program of Korea is for every infant to be vaccinated with BCG within a month after birth; hence, the size of induration as a cutoff diameter of purified protein derivative (PPD) injection site must be greater than $10 \mathrm{~mm}$. During TST, a trained nurse from the KIT intradermally injected $0.1 \mathrm{~mL}$ of PPD test reagent (PPD RT23 2TU, SSI, Copenhagen, Denmark) into the left forearm of each subject. A trained nurse measured the size of the induration using a digital caliper to assess for LTBI positivity 72 hours after the injection. In order to identify LTBI cases using IGRA, all close and casual contacts were encouraged to draw blood for the test. Trained nurses drew 3 $\mathrm{mL}$ of blood from a vein to test with IGRA reagent. QFT-GIT, which determines blood reaction to the specific antigen of Mycobacterium tuberculosis, was used to obtain the result. In this test, effector T lymphocytes secreted serum interferon $\gamma$, and enzyme-linked immunosorbent assay was performed. We adopted the Centers for Disease Control and Prevention (CDC)'s guideline that recommends the cutoff value of 0.35 $\mathrm{IU} / \mathrm{mL}^{18}$. If a subject tested negative in the first round of TST, we performed the second round of TST 8 weeks after the first test. Subjects who underwent second round of TST were diagnosed as having LTBI. When identified as having LTBI by TSTs or QFT-GIT, subjects were advised to undergo preventive chemotherapy by isoniazid or rifampin according to the national guidelines $^{3,15}$.

\section{Statistical analysis}

We analyzed the data using the SAS statistical software package version 9.3 (SAS Institute Inc., Cary, NC, USA). A descriptive analysis was performed using Fisher exact test to determine TST- and IGRA-positive proportion of the contact and the control group. We performed a multivariate logistic regression analysis to determine the significant association between clinical and environmental factors and LTBI among contacts. $\mathrm{p}<0.05$ was considered to be statistically significant, and the $95 \%$ confidence interval was reported to be appropriate.

\section{Ethics statement}

A study protocol in conducting LTBI investigations in military units was reviewed and approved by the Institutional Review Board of the AFMC (AFMC-15-IRB-021). All participants signed the written informed consent form.

\section{Results}

Table 1 shows the proportion of LTBI by the index case. The highest proportion was observed in a military unit with smear $4+$ case and the lowest was in a unit with a non-smear-positive case (Table 1). In all subjects, the mean age of the contact group was 22.5 \pm 5.1 years, and that of the control group was $23.0 \pm 5.3$ years. In both groups, $99 \%$ of all subjects were male. The proportion of subjects having no BCG scar ranges from $11.1 \%$ to $35.7 \%$. In terms of the location of subjects with regard to an index case, $11.0 \%$ of close contacts of smear-positive cases and $22.2 \%$ of close contacts of smear-negative cases reported staying in the same room for greater than 3 months (Table 2).

Table 3 demonstrates the results of LTBI screening test that includes CXR, TST, and QFT-GIT. Only close and casual contacts underwent CXR tests. Three subjects among close contacts of smear-positive cases showing signs of active pulmonary TB infection were referred to military hospitals for further diagnosis and treatment and were excluded from further undergoing other protocols. Out of the total of 953 subjects, 286 subjects who were part of the control group underwent the first round of TST. The results showed that $41.7 \%$ of close contacts of the smear-positive cases were positive and $35.7 \%$ of casual contacts of the smear-positive cases were positive. The proportions of positive cases in close contacts of smear-negative case and the control group were $8.3 \%$ and $16.8 \%$, respectively. As the subjects, except those in the control group, were tested with QFT-GIT to identify the recent TB infection, $16.6 \%$ of close contacts of smear-positive cases tested positive by QFT-GIT and $21.6 \%$ of casual contacts of smearpositive cases tested positive. However, only one subject of a smear-negative case's close contact was tested positive by QFT-GIT (2.8\%). We performed the second round of TST to determine the subjects showing delayed response to PPD by window period. Among the subjects tested, 17 in the close contact and two in the casual contact tested positive. We identified that the overall LTBI rate among contacts screened was $14.7 \%$ (98/667). It can be divided into the following: $21.8 \%$ of close contacts of smear-positive cases, $8.5 \%$ of casual contacts of smear-positive cases, and $2.8 \%$ of close contacts of smearnegative case. Table 4 demonstrates the proportion of LTBI by residence location with regard to the index cases. The LTBI proportion of subjects who shared the same room was the highest, $43.2 \%$, and subjects who stayed in the next room of the index case was $34.0 \%$.

In order to identify the risk factors of LTBI, we performed a multivariate analysis that considers age, number of BCG scars, subject's relative location with an index case, and sputum test of an index case ( $\mathrm{n}=667)$. In the model, a subject's location and sputum smear demonstrated statistical significance. If a subject had stayed with an index case in the same room for greater than 3 months, the probability of having LTBI increased 
Table 1. Clinical information of the index cases and the proportion of LTBI by case

\begin{tabular}{|c|c|c|c|c|c|c|}
\hline \multirow{2}{*}{ Case } & \multirow{2}{*}{ Age (yr) } & \multirow{2}{*}{ Cavity in CXR } & \multirow{2}{*}{ Sputum smear } & \multirow{2}{*}{ Sputum culture } & \multicolumn{2}{|c|}{ No. (\%) } \\
\hline & & & & & Non LTBI & LTBI \\
\hline A & 23 & No & $4+$ & Yes & 29/41 (70.7) & $12 / 41(29.3)$ \\
\hline B & 21 & Yes & $1+$ & Yes & $51 / 55(92.7)$ & 4/55 (7.3) \\
\hline $\mathrm{C}$ & 25 & Yes & $2+$ & Yes & $355 / 400(88.8)$ & $45 / 400(11.3)$ \\
\hline $\mathrm{D}$ & 22 & Yes & $2+$ & Yes & $10 / 13(76.9)$ & $3 / 13(23.1)$ \\
\hline $\mathrm{E}$ & 22 & Yes & $1+$ & Yes & 89/122 (73.0) & $33 / 122(27.0)$ \\
\hline $\mathrm{F}$ & 21 & Yes & - & No & $35 / 36(97.2)$ & $1 / 36(2.8)$ \\
\hline Total & & & & & $569 / 667$ (85.3) & $98 / 667(14.7)$ \\
\hline
\end{tabular}

Values are presented as number (\%).

LTBI: latent tuberculosis infection; CXR: chest X-ray.

Table 2. General characteristics of the study population

\begin{tabular}{|c|c|c|c|c|}
\hline Variable & $\begin{array}{l}\text { Close contacts of } \\
\text { smear positive case } \\
(n=326)\end{array}$ & $\begin{array}{l}\text { Casual contacts of } \\
\text { smear positive case } \\
\qquad(n=305)\end{array}$ & $\begin{array}{c}\text { Close contacts of } \\
\text { smear negative case } \\
(n=36)\end{array}$ & $\begin{array}{l}\text { Control } \\
(n=286)\end{array}$ \\
\hline \multicolumn{5}{|l|}{ Sex } \\
\hline Male & $323(99.1)$ & $305(100)$ & $36(100)$ & $283(99.0)$ \\
\hline Female & $3(0.9)$ & $0(0)$ & $0(0)$ & $3(1.0)$ \\
\hline \multicolumn{5}{|l|}{ Age (yr) } \\
\hline $18-29$ & 305 (93.6) & $281(92.1)$ & $33(91.7)$ & $260(90.9)$ \\
\hline $30-60$ & $21(6.4)$ & $24(7.9)$ & $3(8.3)$ & $26(9.1)$ \\
\hline \multicolumn{5}{|l|}{ BCG scar* } \\
\hline Yes & $242(74.5)$ & $196(64.3)$ & $32(88.9)$ & $211(73.8)$ \\
\hline No & $83(25.5)$ & $109(35.7)$ & $4(11.1)$ & $75(26.2)$ \\
\hline Location (with an index case) & & & & $\mathrm{N} / \mathrm{A}$ \\
\hline Same room & $36(11.0)$ & $0(0)$ & $8(22.2)$ & \\
\hline Next room & $50(15.3)$ & $0(0)$ & $0(0)$ & \\
\hline Opposite room & $87(26.7)$ & $0(0)$ & $0(0)$ & \\
\hline Different floor in the same building & $84(25.8)$ & $0(0)$ & $28(77.8)$ & \\
\hline Different building or commute & $69(21.2)$ & $305(100)$ & $0(0)$ & \\
\hline
\end{tabular}

Values are presented as number (\%).

*For BCG scar, only 325 subjects among close contacts were investigated.

BCG: bacillus Calmette-Guerin; N/A: not applicable.

tenfold. Subjects living in the next room also demonstrate an AOR of 5.19 when controlling other variables. A positive result in the sputum smear test also profoundly influenced the likelihood of having LTBI (Table 5).

\section{Discussion}

This study demonstrated the impact of infectious pulmonary TB cases to contacts in the Korean military settings by reviewing the results of contact investigations in six military units. The overall positive rate of TST was $30.7 \%$ of all close and casual contacts, which was lower than the rate found by Ji et al.'s study in $2007(40.9 \%)^{19}$. The positive rate of TST in the control group was $16.8 \%$, which is similar to the positive rate of TST of military conscripts in 2011, which is $15.4 \%^{20}$. However, this rate was lower than the rate of military medics (19.6\%) who might have been exposed to TB patients in military hospital settings ${ }^{5}$. The overall rate (14.7\%) of LTBI was similar to the rate (14.3\%) identified by a study on TB outbreak 
Table 3. Results of screening for latent tuberculosis infection

\begin{tabular}{|c|c|c|c|c|c|}
\hline & $\begin{array}{c}\text { Close contacts of smear } \\
\text { positive case }\end{array}$ & $\begin{array}{l}\text { Casual contacts of } \\
\text { smear positive case }\end{array}$ & $\begin{array}{c}\text { Close contacts of smear } \\
\text { negative case }\end{array}$ & Control & p-value \\
\hline First TST & & & & & $<0.001$ \\
\hline Negative (<10 mm) & 176/302 (58.3) & 196/305 (64.3) & $33 / 36(91.7)$ & 238/286 (83.2) & \\
\hline Positive ( $\geq 10 \mathrm{~mm}$ ) & $126 / 302(41.7)$ & 109/305 (35.7) & 3/36 (8.3) & 48/286 (16.8) & \\
\hline QFT-GIT & & & & & 0.031 \\
\hline Negative & $271 / 325(83.4)$ & $87 / 111(78.4)$ & $35 / 36(97.2)$ & $\mathrm{N} / \mathrm{A}$ & \\
\hline Positive & $54 / 325(16.6)$ & $24 / 111(21.6)$ & $1 / 36(2.8)$ & $\mathrm{N} / \mathrm{A}$ & \\
\hline Second TST & & & & & $<0.001$ \\
\hline Negative (<10 mm) & $112 / 129(86.8)$ & 145/147 (98.6) & $24 / 24(100)$ & $\mathrm{N} / \mathrm{A}$ & \\
\hline Positive ( $\geq 10$ mm) & $17 / 129(13.2)$ & 2/147 (1.4) & $0 / 24(0)$ & $\mathrm{N} / \mathrm{A}$ & \\
\hline LTBI & & & & & $<0.001$ \\
\hline Negative & $255 / 326(78.2)$ & 279/305 (91.5) & $35 / 36(97.2)$ & $\mathrm{N} / \mathrm{A}$ & \\
\hline Positive & 71/326 (21.8) & 26/305 (8.5) & 1/36 (2.8) & $\mathrm{N} / \mathrm{A}$ & \\
\hline
\end{tabular}

Values are presented as number (\%).

TST: tuberculin skin test; QFT-GIT: QuantiFERON-TB Gold In-Tube; N/A: not applicable; LTBI: latent tuberculosis infection.

Table 4. The proportions of latent tuberculosis infection cases by living location

\begin{tabular}{|lcc|}
\hline \multicolumn{1}{|c|}{ Location with the index cases } & LTBI & No \\
\cline { 2 - 3 } Same room $(\mathrm{n}=44)$ & $25(56.8)$ & $19(43.2)$ \\
\hline Next room $(\mathrm{n}=50)$ & $33(66.0)$ & $17(34.0)$ \\
\hline Opposite room $(\mathrm{n}=87)$ & $75(86.2)$ & $12(13.8)$ \\
\hline Different floor in the same building $(\mathrm{n}=112)$ & $93(83.0)$ & $19(17.0)$ \\
\hline Different building or commute $(\mathrm{n}=374)$ & $343(91.7)$ & $31(8.3)$ \\
\hline Total $(\mathrm{n}=667)$ & $569(85.3)$ & $98(14.7)$ \\
\hline
\end{tabular}

Values are presented as number (\%).

LTBI: latent tuberculosis infection.

investigations in 2007 using IGRA ${ }^{11}$. The similarity of LTBI may suggest that the environments in the Army units have not been significantly modified in terms of preventing the spread of respiratory infectious diseases especially TB. With information on age, BCG scar, a subject's location with regard to an index case, and sputum smear test, we performed a multivariate logistic regression analysis to identify and assess the risk factors. It showed that living in the same room or next room is significantly associated with LTBI, AOR of 10.93 and 5.19, respectively.

The military tuberculosis guideline in 2012 defined a contact as a person who has shared the same room with a pulmonary TB patient for greater than 3 months ${ }^{15}$. In addition to this definition, the recent national tuberculosis treatment guideline defines a close contact as a person who shares a closed space for a long duration such as greater than 40 hours with a pulmonary TB patient ${ }^{3}$. However, those guidelines also recommend that in defining a close contact, the justifications made by medical epidemiologists who conduct a contact investigation should be considered. In practice, military epidemiologists need to guide a military commander who is in charge of the unit to conduct an investigation and to ensure all military personnel who are at risk of TB exposure are included in the contact investigation. In this regard, we anticipate that the findings of this study contribute in properly defining close and casual contacts in the military context.

In this study, we address confined living settings and close proximity between military individuals who contribute to the transmission of TB. It has been highlighted that respiratory diseases such as TB are likely to spread among military recruits due to the unique environment in the military units ${ }^{21}$. In the U.S. military, there have been a number of reports on TB outbreaks in Naval ships and Marine units where only very confined space is provided to military individuals ${ }^{22,23}$. A Swiss 
Table 5. Multivariable logistic regression test for tuberculin skin test and latent tuberculosis infection

\begin{tabular}{|c|c|c|}
\hline \multirow[t]{2}{*}{ Variable } & \multicolumn{2}{|c|}{$\begin{array}{c}\text { Latent tuberculosis } \\
\text { infection* }\end{array}$} \\
\hline & AOR & $95 \% \mathrm{CI}$ \\
\hline \multicolumn{3}{|l|}{ Age (yr) } \\
\hline$<30$ & Ref. & \\
\hline$\geq 30$ & 0.21 & $0.03-1.57$ \\
\hline \multicolumn{3}{|l|}{ BCG scar on shoulder } \\
\hline Yes & Ref. & \\
\hline No & 1.13 & $0.68-1.88$ \\
\hline \multicolumn{3}{|l|}{ Sputum smear of the index case } \\
\hline \multicolumn{3}{|l|}{ Control } \\
\hline Negative & Ref. & \\
\hline $1+$ & 14.34 & $1.84-111.89$ \\
\hline $2+$ & 16.01 & $1.93-132.98$ \\
\hline $4+$ & 22.63 & $2.48-206.18$ \\
\hline \multicolumn{3}{|l|}{ Location (with the index cases) } \\
\hline Different building or commute & Ref. & \\
\hline Different floor in a same building & 3.43 & $1.39-8.47$ \\
\hline Opposite room & 1.54 & $0.71-3.32$ \\
\hline Next room & 5.19 & $2.34-11.52$ \\
\hline Same room & 10.93 & $4.93-24.25$ \\
\hline
\end{tabular}

*The statistical model for LTBI positive that includes age, BCG scar, sputum smear results and location with the index case as variables analyzed data of 446 subjects who underwent IGRA test.

AOR: adjusted odds ratio; CI: confidence interval; Ref.: reference value; BCG: bacillus Calmette-Guerin; LTBI: latent tuberculosis infection; IGRA: interferon $\gamma$ release assay.

report on LTBI shows the effect of communal settings on the spread of TB infection ${ }^{24}$. Military conscripts who shared the same dormitory with pulmonary TB patients displayed a LTBI rate of $94 \%$ (14/15), but other groups who never shared the same living place with pulmonary TB patients showed a LTBI rate in the range of 9.9\%-22.7\%. Fujikawa et al. ${ }^{25}$ reported the outcomes of a contact investigation with 884 ground selfdefense force members using IGRA, CXR, and computed tomography. It was found that more than one-third of subjects $(39 / 108,36.1 \%)$ in the LTBI group had resided on the same floor in the barrack with pulmonary TB patients.

In Korea, more than 30,000 new TB cases every year were identified, and TB caused 2,209 deaths in $2015^{1-3}$. Despite the Ministry of Health and Welfare and KCDC implementing TB management programs under the strengthened new TB management policies that include minimizing treatment failure, mandatory inpatient treatment in case of multidrug-resistant $\mathrm{TB}$, and enhanced contact management, the TB notification rate still remains at 55.0 per 100,000 population ${ }^{1}$. A study that reviewed $\mathrm{TB}$ investigation data suggested that recent $\mathrm{TB}$ outbreaks were focused on congregate settings such as in the nurseries, schools, military or police units, and correctional facilities $^{17}$. In the study, military or police units account for $11.6 \%$ of LTBI cases, following correctional facilities and workplaces. It emphasizes the importance of TB investigation particularly for population living and working in congregate settings such as the military. This study therefore has an implication that it assessed the results of multiple TB contact investigations in Korean military settings. It offers valuable information in defining contacts by measuring the impact of environmental risk factor. In order to minimize the impact of an infectious TB case within a military unit, there is a demand to enhance soldiers' knowledge and attitude toward the prevention of TB through education. There has been no military-wide survey assessing the Army soldiers' knowledge and attitude, but a recent survey conducted with 500 male Army soldiers shows a lack of knowledge on TB among the Army soldiers ${ }^{26}$.

However, we need to note the limitations of this study. First, this study was designed and conducted to include only the Army units. This limits the generalizability of the findings as the living environment of the Navy and Air Force units is different from that of the Army units. The detailed information of military units is classified and has not been disclosed to the public, but we suspect that the more congregate environment of the Army units has a significant impact on the transmission of communicable diseases including TB. Confirming this hypothesis requires additional information to compare the physical environment of the services. Second, this study missed a portion of the study subjects due to the subjects' transfer, leave, and military drill. We could not include 17 close contacts, 163 casual contacts for the first round of TST, and 191 close contacts among the 357 subjects tested for TST and QFT-GIT. This high proportion of missing subjects may have contributed to the inaccurate rates for comparability with the other studies. Third, the design of this study did not include follow-up steps to identify subsequent TB cases in the study sites. During the study, we identified three cases of suspected active TB cases with the first CXR and another three cases by the second CXR. However, we were not able to follow up the military units regarding the presence of subsequent $\mathrm{TB}$ cases due to the index cases, hence limiting the assessment of a long-term effect of TB to a population where frequent transfer and move occur. It highlights a need to design a comprehensive study that integrates the military health systems and public health systems such as public health centers and KCDC where all TB cases are notified for treatment and prevention ${ }^{15}$. Fourth, the positive reaction in the second round of TST may have been caused by the recall reaction by the previous BCG vaccination. However, in the close contacts of smearnegative index cases, all the 24 subjects who had undergone the second round of TST were negative. Therefore, most of the 
positive reactors of the smear-positive contact group are more likely to be infected with Mycobacterium tuberculosis. Fifth, the study could not provide further information on why the subjects who reported that they stayed in the opposite room show nonsignificant AOR. Additionally, a group of subjects who reported staying in a different floor in the same building was found to have significant AOR (3.43, 1.39-8.47). This indicates that further research should confirm this finding with a larger number of subjects in the same study environment.

In conclusion, we analyzed the data from $\mathrm{TB}$ contact investigations in the Korean Army from September 2012 to May 2013 to identify the impact of communal living and other risk factors. By performing TST and QFT-GIT, we identified an overall LTBI rate of 14.7\% (98/667) among close and casual contacts screened. A multivariate analysis demonstrated that living in the same room or next room is significantly associated with LTBI (AOR in the same room, 10.93; AOR in the next room, 5.19). Our study demonstrated a significant impact of communal living in LTBI when exposed to a TB case and provided evidence on how to define the scope of close and casual contacts of an index TB case in Korean Army settings.

\section{Authors' Contributions}

Conceptualization: Kim HJ, Kang DY, Yoon CG, Oh SY, Lee JB, Kim MH, Seo Y. Methodology: Kim HJ, Kang DY, Yoon CG, Oh SY, Lee JB, Kim MH, Seo Y. Formal analysis: Kim HJ, Kang DY, Yoon CG. Writing - original draft preparation: Kim HJ, Jung J, Yoon CG. Writing - review and editing: Kim HJ, Jung J, Yoon CG. Approval of final manuscript: all authors.

\section{Conflicts of Interest}

No potential conflict of interest relevant to this article was reported.

\section{Funding}

This project was supported by the research fund of the Armed Forces Medical Research Institute.

\section{References}

1. Korea Centers for Disease Control and Prevention. 2017 Annual report on the notified tuberculosis in Korea. Cheongju: Korea Centers for Disease Control and Prevention; 2018.

2. Statistics Korea. Causes of death statistics in 2015. Daejeon: Statistics Korea; 2016.

3. Korea Centers for Disease Control and Prevention. National
Tuberculosis Control Program Guideline. Cheongju: Korea Centers for Disease Control and Prevention; 2018.

4. Kim SJ, Bai GH, Lee H, Kim HJ, Lew WJ, Park YK, et al. Transmission of Mycobacterium tuberculosis among high school students in Korea. Int J Tuberc Lung Dis 2001;5:824-30.

5. Yoon CG, Oh SY, Lee JB, Kim MH, Seo Y, Yang J, et al. Occupational risk of latent tuberculosis infection in health workers of 14 military hospitals. J Korean Med Sci 2017;32:1251-7.

6. Park JS. The prevalence and risk factors of latent tuberculosis infection among health care workers working in a tertiary hospital in South Korea. Tuberc Respir Dis 2018;81:274-80.

7. Kang CI, Choi CM, Kim DH, Kim CH, Lee DJ, Kim HB, et al. Pulmonary tuberculosis in young Korean soldiers: incidence, drug resistance and treatment outcomes. Int J Tuberc Lung Dis 2006;10:970-4.

8. Lin JC, Lin TY, Perng WC, Mai CS, Chen YH, Ku CH, et al. An outbreak of tuberculosis in a bacillus Calmette-guerin-vaccinated military population. Mil Med 2008;173:388-92.

9. Lee SW, Jang YS, Park CM, Kang HY, Koh WJ, Yim JJ, et al. The role of chest CT scanning in TB outbreak investigation. Chest 2010;137:1057-64.

10. Lee SW, Jeon K, Kim KH, Min KH. Multidrug-resistant pulmonary tuberculosis among young Korean soldiers in a communal setting. J Korean Med Sci 2009;24:592-5.

11. Choi CM, Hwang SS, Lee CH, Lee HW, Kang CI, Kim CH, et al. Latent tuberculosis infection in a military setting diagnosed by whole-blood interferon-gamma assay. Respirology 2007;12:898-901.

12. Choi CM, Kang CI, Jeung WK, Kim DH, Lee CH, Yim JJ. Role of the C-reactive protein for the diagnosis of TB among military personnel in South Korea. Int J Tuberc Lung Dis 2007;11:2336.

13. Yoon H, Jhun BW, Kim SJ, Kim K. Clinical characteristics and factors predicting respiratory failure in adenovirus pneumonia. Respirology 2016;21:1243-50.

14. Yoo H, Gu SH, Jung J, Song DH, Yoon C, Hong DJ, et al. Febrile respiratory illness associated with human adenovirus type 55 in South Korea Military, 2014-2016. Emerg Infect Dis 2017;23:1016-20.

15. Ministry of National Defense; Korea Centers for Disease Control and Prevention. Military tuberculosis control manual. Cheongju: Korea Centers for Diseases Control and Prevention; 2012

16. Choi CM, Kang CI, Kim DH, Kim CH, Kim HJ, Lee CH, et al. The role of TST in the diagnosis of latent tuberculosis infection among military personnel in South Korea. Int J Tuberc Lung Dis 2006;10:1342-6.

17. Kwon Y, Kim SJ, Kim J, Kim SY, Song EM, Lee EJ, et al. Results of tuberculosis contact investigation in congregate settings in Korea, 2013. Osong Public Health Res Perspect 2014;5(Suppl):S30-6.

18. Mazurek GH, Jereb J, Vernon A, LoBue P, Goldberg S, Castro $\mathrm{K}$, et al. Updated guidelines for using interferon gamma re- 
lease assays to detect Mycobacterium tuberculosis infection: United States, 2010. MMWR Recomm Rep 2010;59:1-25.

19. Ji SH, Kim HJ, Choi CM. Management of tuberculosis outbreak in a small military unit following the Korean National Guideline. Tuberc Respir Dis 2007;62:5-10.

20. Lee SW, Oh SY, Lee JB, Choi CM, Kim HJ. Tuberculin skin test distribution following a change in BCG vaccination policy. PLoS One 2014;9:e86419.

21. Kak V. Infections in confined spaces: cruise ships, military barracks, and college dormitories. Infect Dis Clin North Am 2007;21:773-84.

22. Lamar JE 2nd, Malakooti MA. Tuberculosis outbreak investigation of a U.S. Navy amphibious ship crew and the Marine expeditionary unit aboard, 1998. Mil Med 2003;168:523-7.
23. Foote FO. A tuberculosis event on a Navy assault ship. Mil Med 2006;171:1198-200.

24. Kipfer B, Reichmuth M, Buchler M, Meisels C, Bodmer T. Tuberculosis in a Swiss army training camp: contact investigation using an interferon gamma release assay. Swiss Med Wkly 2008;138:267-72.

25. Fujikawa A, Fujii T, Mimura S, Takahashi R, Sakai M, Suzuki $\mathrm{S}$, et al. Tuberculosis contact investigation using interferongamma release assay with chest $\mathrm{x}$-ray and computed tomography. PLoS One 2014;9:e85612.

26. Choi Y, Jeong GH. Army soldiers' knowledge of, attitude towards, and preventive behavior towards tuberculosis in Korea. Osong Public Health Res Perspect 2018;9:269-77. 